\title{
Virtual character facial expressions influence human brain and facial EMG activity in a decision-making game
}

\section{Ravaja, Niklas}

2018-04

Ravaja , N , Bente , G , Kätsyri , J , Salminen , M \& Takala , T 2018 , ' Virtual character facial expressions influence human brain and facial EMG activity in a decision-making game ' , IEEE Transactions on Affective Computing , vol. 9 , no. 2 , pp. 285-298 . https://doi.org/10.1109/TAFFC.2016.26011

http://hdl.handle.net/10138/232744

https://doi.org/10.1109/TAFFC.2016.2601101

acceptedVersion

Downloaded from Helda, University of Helsinki institutional repository.

This is an electronic reprint of the original article.

This reprint may differ from the original in pagination and typographic detail.

Please cite the original version. 


\title{
Virtual Character Facial Expressions Influence Human Brain and Facial EMG Activity in a Decision-Making Game
}

\author{
Niklas Ravaja, Gary Bente, Jari Kätsyri, Mikko Salminen, and Tapio Takala
}

\begin{abstract}
We examined the effects of the emotional facial expressions of a virtual character (VC) on human frontal electroencephalographic (EEG) asymmetry (putatively indexing approach/withdrawal motivation), facial electromyographic (EMG) activity (emotional expressions), and social decision making (cooperation/defection). In a within-subjects design, the participants played the Iterated Prisoner's Dilemma game with VCs with different dynamic facial expressions (predefined or dependent on the participant's electrodermal and facial EMG activity). In general, VC facial expressions elicited congruent facial muscle activity. However, both frontal EEG asymmetry and facial EMG activity elicited by an angry VC facial expression varied as a function of preceding interactional events (human collaboration/defection). Pre-decision inner emotional-motivational processes and emotional facial expressions were dissociated, suggesting that human goals influence pre-decision frontal asymmetry, whereas display rules may affect (pre-decision) emotional expressions in human-VC interaction. An angry VC facial expression, high pre-decision corrugator EMG activity, and relatively greater left frontal activation predicted the participant's decision to defect. Both post-decision frontal asymmetry and facial EMG activity were related to reciprocal cooperation. The results suggest that the justifiability of $\mathrm{VC}$ emotional expressions and the perceived fairness of $\mathrm{VC}$ actions influence human emotional responses.
\end{abstract}

Index Terms - Virtual characters, emotions, neurophysiology, decision making

\section{INTRODUCTION}

$\mathrm{T}$ HE role of emotions in human decision making is well established [1], [2], [3], [4]. When humans make decisions whether to trust and cooperate with others, they usually rely on different types of information, including the action history of the other (if available [5]) and tacit cues of trustworthiness derived from physical appearance [6], [7], and, more importantly, nonverbal emotional expressions [8], [9]. Recent research shows that also emotional facial expressions of a VC (or embodied agent) affect human decision making in human-VC interaction [10], [11], [12]. For example, de Melo and colleagues found that people concede more to an agent that expresses anger compared to an agent expressing happiness in human-agent negotiation [10], and cooperate more with an agent communicating a cooperative vs. individualistic goal orientation through facial display patterns [11].

The interpretation of nonverbal behavior is strongly dependent on the context (e.g., a smile after having defected may be interpreted differently compared to a smile after having cooperated [13]). However, and even though the influence of VC emotional displays on decision making is likely to be mediated by human emotions, there is a

- N. Ravaja is with the Helsinki Collegium for Advanced Studies, University of Helsinki, and School of Business, Aalto University, Finland. E-mail: niklas.ravaja@helsinki.fi.

- G. Bente is with the University of Cologne, Germany. E-mail: gabente@gmx.de.

- J. Kätsyri is with the School of Science, Aalto University, Helsinki, Finland. E-mail: jari.katsyri@aalto.fi.

- M. Salminen is with the School of Business, Aalto University, Helsinki, Finland. E-mail: mikko.salminen@aalto.fi.

- T. Takala is with the School of Science, Aalto University, Helsinki, Finland. E-mail: tapio.takala@aalto.fi. paucity of studies examining human emotionalmotivational processes, in particular at the level of brain activities and facial expressions, elicited by VC facial displays in different contexts (e.g., preceding interactional events; but see [14]). Therefore, the present study was designed to examine how the facial displays of a VC influence human approach/withdrawal motivation, as indexed by electroencephalographic (EEG) asymmetry over the prefrontal cortex (relative activity of the left and right hemispheres), and emotional expressions, as indexed by facial electromyography (EMG), during the Iterated Prisoner's Dilemma game (with monetary or equivalent stakes). We expected that inner emotional-motivational processes (putatively indexed by frontal EEG asymmetry) and emotional facial expressions may be dissociated, assuming that display rules (i.e., learned rules dictating the management of emotional expressions based on social circumstances) might affect emotional expressions also in human-VC interaction (see e.g., [15]). We then also examined how frontal EEG asymmetry and facial EMG activity predict cooperation in the game, and how the outcome of the game affects physiological responses.

\subsection{VC's Facial Expressions and Decision Making}

There are two main theoretical positions on the role of facial expressions. According to the emotion-expression view, facial displays are first and foremost readouts of a person's internal emotional state (e.g., [16]). According to the behavioral ecology view, facial displays are social signals, communicating behavioral intentions or social motives [17]. However, recent evidence indicates that, in so- 
cial situations, displays are affected by both emotional and social factors (e.g., [18], [19], see also [80]; for a review, see [20]). Appraisal theories posit that emotions are elicited and differentiated by the results of the individual's evaluation of events in terms of their consequences for one's goals or needs [21].

In many situations, people have to choose whether to pursue their own self-interest or to rely on another person to maximize collective interests [22]. Cooperation may entail gains, but there is a risk of being exploited by cheaters taking advantage of the cooperator [23]. Trust plays an important role in the development of cooperation [24]. Clearly, for an individual, it is advantageous to be able to quickly detect interaction partners whom one can trust and who are likely to be cooperative. Cooperative and altruistic individuals have been shown to display higher levels of positive emotion compared to noncooperators, although the degree to which displays of positive emotion reflect a cooperative disposition may depend on the situation (e.g., [8]). However, Schug, Matsumoto, Horita, Yamagishi, and Bonner [9] suggested that overall emotional expressivity may be a more reliable signal of cooperativeness than displays of positive emotion alone. Photographs of smiling faces of alleged non co-located humans have also been shown to elicit cooperation from participants in a trust game [25]. The interpretation of a smile in terms of the intention it indicates is also modulated by interpersonal factors, such as in-group and out-group membership [79]. This conforms to the view that emotional expressions provide information about a person's current emotions, beliefs, intentions, and orientations towards the relationship (e.g., trustworthy or antagonistic [26], [27]). Human decision making is influenced by emotional expressions of not only other humans but also VCs. For example, de Melo and co-workers [28] have shown that people cooperate more with a VC expressing moral emotions (anger, shame, gratitude) according to the action history of the Iterated Prisoner's Dilemma game compared to a VC expressing no emotions (see also [11]).

According to Frank's [29] view, before engaging in cooperation in social dilemmas, people look for cues in others that they might be willing to cooperate. Hence, one would expect that happy (smiling) VC faces would elicit cooperation in the Iterated Prisoner's Dilemma game. VC cooperation in the previous trial may also increase the confidence in the benevolent social meaning of a smile. In contrast, an angry face is a threatening stimulus that would be expected to elicit distrust [30, see also 78]. People may infer that a person expressing anger is not likely to engage in cooperative behavior. This leads to the first hypothesis $(\mathrm{H})$ :

H1: A happy VC facial expression will elicit cooperation, especially when the VC has cooperated in the previous trial, whereas an angry VC facial expression will elicit defection.

\subsection{Human Facial Expressions during Human- Virtual Character Interaction}

Given the role played by emotional displays in engaging in cooperation, it is also of import to examine the factors influencing human facial expressions when interacting with a VC. Humans are predisposed to react to emotional facial expressions with facial mimicry in terms of specific, congruent facial muscle activity [31]. Studies using facial electromyography (EMG) have shown that happy facial stimuli spontaneously evoke increased activity over zygomaticus major (cheek muscle region that is activated when smiling) and orbicularis oculi (periocular muscle region that is activated particularly during enjoyment smile), whereas angry faces elicit increased activity over corrugator supercilii (brow muscle area that is activated during frowning [31], [32], [33]. Facial reactions have been suggested as being controlled by biologically given affect programs that operate automatically and independently of any conscious cognitive processes [31]. Observing another person's facial expression may also induce a similar emotional state in the observer (i.e., emotional contagion [34]). Not only human emotional facial expressions but also VC facial expressions elicit congruent facial muscle activity. In two studies, a VC's happy expression elicited increased zygomatic EMG activity, but an angry expression elicited no significant corrugator supercilii activation [30], [35]. Hence, we presented the following hypothesis:

$\mathrm{H} 2$ : Happy and (pleasantly) relaxed VC faces will elicit greater zygomaticus major and orbicularis oculi, and lower corrugator supercilii, facial EMG activity compared to a neutral face, whereas the opposite will be true for angry and sad VC faces.

Although facial EMG measures may be related to a person's internal emotional state (for a review, see [36]), we expected that human facial expressions as measured by facial EMG would primarily serve a social communicative function when interacting with a VC in the Iterated Prisoner's Dilemma game. No prior studies have yet examined whether display rules affecting emotional facial expressions in human-to-human interaction will also affect human facial expressions in human-VC interaction. People have been suggested to learn, through a process of socialization, to manage the appearance of particular emotions in particular situations ([37], [15]). That is, people may inhibit (deintesify) or facilitate (intensify) their facial behavior in accordance with their knowledge of the appropriateness or advantageousness of a particular emotional expression in a particular context. The application of display rules can also occur automatically and outside of awareness [15]. We expected that human (pre-decision) facial expressions elicited by VC facial displays would depend also on the preceding interactional events (e.g., human cooperation). Anger has been suggested to serve as a call for behavioral adjustment [38]. To safeguard successful social exchange, it is usually wise to take into account the expression of anger by one's interaction partner. Thus, a VC angry expression after the participant has defected (noncooperated) should lead to diminished smiling (as smiling could be interpreted as a sign of malicious pleasure) and diminished frowning (as the VC anger expression can be regarded as justifiable) by the participant. This leads to the next hypothesis.

H3: An angry VC facial expression will elicit less zy- 
gomatic, orbicularis oculi, and corrugator EMG activity (less smiling and frowning) when the participant has defected compared to cooperated in the previous trial.

As noted above, facial expressions may provide information on intentions and future actions [17]. A frowning expression (typical also for anger) would be expected to signal antagonism rather than willingness to engage in cooperative behavior. Pillutla and Murnighan [39] also showed that anger was a better predictor of rejections than unfairness perceptions in the ultimatum game. This leads to $\mathrm{H} 4$ that is complementary to $\mathrm{H} 1$ :

H4: High corrugator supercilii EMG activity during the pre-decision period will predict defection by the participant.

\subsection{Inner Emotional-Motivational Processes during Human-Virtual Character Interaction}

We expected that human inner emotional-motivational processes may differ from emotional facial expressions in human-VC interaction and would primarily be related to the anticipation of (monetary or equivalent) rewards (i.e., one's goals) in the decision-making game. According to the approach-withdrawal motivational model of emotion, the left- and right-anterior regions of the brain are part of two separate neural systems underlying approach and withdrawal motivation, respectively (e.g., [40], [41]). Relatively greater left frontal activity, either as a trait or a state, may indicate a propensity to approach or engage a stimulus and is associated with approach-motivated affective states (e.g., joy, desire, enthusiasm, and anger; for reviews, see [42], [40]; [43]). In contrast, relatively greater right frontal activity may indicate a propensity to withdraw or disengage from a stimulus and is associated with withdrawal-motivated affective states (e.g., disgust and fear).

Increased resting left-lateralized activity has also been associated with a stronger bias to respond to (monetary) reward-related cues [44], and resting-state hypoactivity in the right lateral PFC has been shown to predict higher monetary risk taking [45] and a lower willingness to punish in the ultimatum game [46]. Source localization of frontal EEG asymmetry in the alpha frequency band (8-12 $\mathrm{Hz}$; i.e., the index of asymmetrical activation of leftfrontal versus right-frontal brain regions) has indicated that it reflects activity in the dorsal prefrontal cortex (PFC [44]).

The emotion contagion hypothesis predicts that the facial display of emotion evokes a similar (inner) emotional state in the interaction partner [47]. Accordingly, for example, VC expression of anger might be expected to elicit greater relative left frontal activation, given that anger is an approach-related affective state, although it is negative in valence (Harmon-Jones, 2003); relevant to this, relatively greater left frontal activity has been associated with both trait anger and insult-induced state anger [48], [49]. However, frontal EEG asymmetry has primarily been suggested to be associated with pre-goal attainment emotion elicited while attempting to achieve a goal (e.g., enthusiasm), but not with post-goal attainment emotion (e.g., contentment [50]). Thus, we expected that, in the context of the Prisoner's Dilemma game, people focus on the salient goal of achieving rewards, and pre-decision frontal asymmetry would primarily be related to anticipated rewards (rather than to anger directly elicited by an angry VC expression). Given that people are likely to infer that an angry $\mathrm{VC}$ is antagonistic and reluctant to cooperate, making it impossible to get a high payoff (payoff matrix is known), people may anticipate nonreward (associated with reduced approach motivation) when the VC is angry (VC anger serves as a cue of nonreward). This leads to $\mathrm{H} 5$ :

H5: An angry VC face will elicit diminished (predecision) relative left frontal activation.

Apparently, people wish and expect that their cooperation will be reciprocated with cooperation by others [23]. Thus, the expression of anger by one's interaction partner in response to cooperation may be a particularly prominent and disappointing cue of forthcoming nonreward. That being so, we predicted the following:

H6: An angry (but not neutral) VC will elicit less (predecision) relative left frontal activation when the participant has cooperated compared to defected in the previous trial.

Pre-decision frontal asymmetry may also predict cooperation/defection. The pursuit for a high payoff (accompanied by high approach motivation) necessitates defection by the participant. This leads to the next hypothesis:

H7: Relatively greater left frontal activation will predict defection.

\subsection{Post-Decision Facial Expressions and Frontal Asymmetry}

An important question is whether post-decision emotional facial expressions and frontal asymmetry are primarily determined by the payoffs (obtained rewards) or reciprocated cooperation, or lack thereof. Achieving one's goal to get a high payoff would be expected to elicit positive affect, including positive facial expressions [51]. However, it is also possible that reciprocal cooperation evokes positive emotional expressions. Frontal asymmetry should not be associated with post-goal attainment emotion [50] but reciprocal cooperation could be expected to elicit approach motivation. Despite these possible differential predictions, we presented the following hypothesis:

H8: Post-decision zygomatic and orbicularis oculi EMG activity will increase, and corrugator activity will decrease, as a function of payoffs, and greater relative left frontal activation will be related to reciprocal cooperation.

\subsection{Responsive VC Facial Expressions}

As noted above, in human-to-human interaction, the display of emotions elicits complementary or similar empathic emotions in others [34]; being sensitive to others' affect may be adaptive (e.g., it facilitates the smoothness of social interaction [47]). The principle of similarityattraction also posits that individuals are attracted to other people who are similar to themselves (e.g., in terms of personality or dispositional emotionality), and the phenomenon is present also for computer-generated speech manifesting personality [52]. This leads to our secondary 
research question: Does the congruency between VC (predecision) emotional expression and the participant's emotional state (as assessed in real-time on the basis of facial EMG and electrodermal activity, EDA) influence physiological responses and cooperation/defection?

\section{Methods}

\subsection{Participants}

Participants were 37 (10 white male and 27 white female) Finnish undergraduates with various majors, who ranged in age from 20 to 35 years (mean $=24.5$ years). Each participant received two movie tickets for participation and, depending on the outcome of the Iterated Prisoner's Dilemma game, up to three additional movie tickets (see below). Given that eight participants adopted the strategy to always defect in the game (actually the most profitable strategy), their decision making data were excluded from the statistical analyses. In addition, one participant was excluded owing to problems in data collection. Consequently, 28 participants $(8$ men) were included in the analyses of the physiological and decision making data.

\subsection{Design}

An 8 (Facial Expression of a VC: angry, happy, sad, pleasantly relaxed, neutral, no-face, congruent, incongruent) $\times$ 2 (VC: man, woman) $\times 2$ (VC Decision: cooperate, defect) $\times 6$ (Repetition) within-subjects design was employed.

\subsection{Virtual Character}

There were two VCs, one female (called Kata) and one male (called Mikko). The 3D model was created with Blender software (http://www.blender.org), using pictures of a real human female and male as reference pictures. The Blender Game Engine was used for the realtime presentation of the VCs and, together with scripts written in the Python programming language (http://www.python.org), carrying out the experiment (https: / / github.com/MikkoSalminen/tutorial/tree/mast er/Avatar). Given that, in the congruent expression and incongruent expression conditions, the VC facial expressions were dependent on psychophysiological measures of valence (facial EMG) and arousal (EDA; see below), the facial expressions of the VC were intended to correspond theoretically to the four quadrants of the affective space defined by valence and arousal [53]: angry (negative valence and high arousal), happy (positive valence and high arousal), sad (negative valence and low arousal), and pleasantly relaxed (positive valence and low arousal; see Figure 1). In addition, a neutral facial expression was included. Open-mouth angry, open-mouth happy, sad, and neutral expressions were created following Ekman and Friesen's [37] descriptions and using images of prototypical facial expressions from Ekman and Friesen's [54] Pictures of Facial Affect as examples; these facial pictures have previously been judged to be clear examples of the targeted emotions [55]. Given the lack of a prototype corresponding to a pleasantly relaxed face, the pleasantly relaxed facial expression was created with the help of a certified Facial Action Coding System (FACS) coder; the

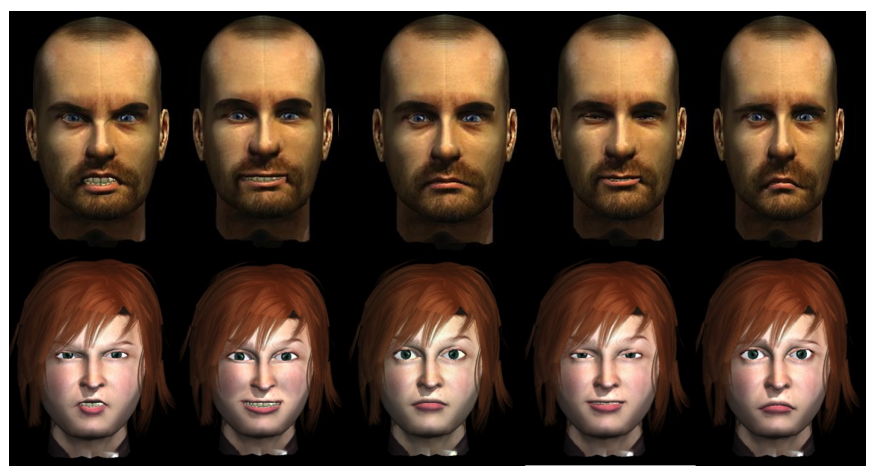

Fig. 1. Male (top row) and female (bottom row) VC facial expressions (from left to right: angry, happy, neutral, positively relaxed, sad).

eyes of the VC were half-closed and the mouth was in a slight smile and slightly ajar to express pleasant relaxation, calmness, and serenity. In each trial, the $\mathrm{VC}^{\prime} \mathrm{s}$ facial expression was initially neutral but after $1 \mathrm{~s}$ dynamically changed into the targeted expression (or remained neutral when the targeted expression was neutral). During the trials, the VC moved its head slowly and its eyes blinked occasionally (for example animations, see supplementary material).

\subsection{Responsive VC Facial Expressions}

To address the secondary research question, in two conditions (i.e., congruent expression and incongruent expression), the facial expressions of the $\mathrm{VC}$ were dependent on the emotional state of the participant as assessed in realtime by facial EMG (putatively indexing emotional valence) and EDA (indexing emotional arousal). In the congruent expression condition, the VC facial expression was happy, relaxed, angry, and sad when the participant's emotional state was assessed as being high-arousal positive, low-arousal positive, high-arousal negative, and low-arousal negative, respectively. In the incongruent expression condition, the VC facial expression was relaxed, happy, sad, and angry when the participant's emotional state was assessed as being low-arousal negative, high-arousal negative, low-arousal positive, and higharousal positive, respectively. One of the four aforementioned VC facial expressions was shown only when both the valence- and arousal-related criteria were met; otherwise the VC facial expression remained neutral. In the beginning of the experiment, there was an EMG calibration period. The participant was asked not to speak and to have a neutral facial expression for $10 \mathrm{~s}$-the first $6 \mathrm{~s}$ were used for calculating mean values for unrectified $z y-$ gomatic, orbicularis oculi, and corrugator EMG. Then, the participant was asked to smile and to frown to provide maximum values (these values were updated continuously throughout the experiment) and to ensure that the participant's facial expressions were recognized correctly by the system. During the experiment, EMG values were rectified in relation to the mean of the calibration period (i.e., values below the mean were mirrored so that all values were above the mean), resampled at $5 \mathrm{~Hz}$, and scaled from 0 (mean of the calibration period) to 100 (maximum value so far). EDA values were updated at $2.5 \mathrm{~Hz}$ and 
compared to the mean of a preceding 20-s window. The valence- and arousal-related criteria, determined on the basis of pre-tests, were the following: (a) for positive valence, (zygomatic EMG + orbicularis oculi EMG) / $2>5.2$, (b) for negative valence, corrugator EMG $>2.25$ and $z y-$ gomatic EMG $<1.5$, (c) for high arousal, EDA $>1.125 \times$ mean EDA during the preceding 20-s window, and (d) for low arousal, EDA $\leq 1.125 \times$ mean EDA during the preceding 20-s window. Using these criteria, an emotional-state classification was made during the second preceding the presentation of the $\mathrm{VC}$, which determined the $\mathrm{VC}$ facial expression for that trial.

\subsection{Procedure}

In the laboratory, the participant was first given instructions on the task and tested for task comprehension. After the briefing, the participant filled out an informed consent form. Electrodes were then attached, and the participant was seated on a chair and left alone in the laboratory (an electrically shielded sound-attenuated room) for an 8-min rest period. Then there was an EMG calibration period for the real-time emotion assessment (see Responsive VC Facial Expressions and Physiological Data Collection), followed by the experiment that took, on the average, 62 min. The participant played the Iterated Prisoner's Dilemma game with the two computer-controlled VCs whose facial expressions varied. The conditions were angry, happy, sad, pleasantly relaxed, neutral, no-face (VC was not shown), congruent expression (emotional expression corresponding to the participant's emotional state), and incongruent expression (emotional expression opposite to the participant's emotional state). In each trial, the participant and the VC decided either to collaborate or not (i.e., defect; the decisions of the VC were actually predefined). The players received points as follows: (a) if both players chose to collaborate, both received three points, (b) if both players chose to defect, both received one point, and (c) if one player chose to collaborate and the other one chose to defect, the former received zero points and the latter five points. The decisions by the players and the points received by both players were shown after the participant had made his or her decision. The participant was told that there would be several trials, but the exact number of trials was not revealed in advance.

To ensure the participant's engagement in the game, the participant was told that he or she will get additional movie tickets depending on his or her total score in the game as follows: $400-449$ points $=$ one movie ticket (value of $10 €), 450-499$ points = two movie tickets, and over 500 points $=$ three movie tickets. The total score of the participants (who were included in the statistical analyses) ranged from 396 to $554(M=487, S D=37)$.

All participants were presented with 192 trials in a random order. Each of the trials consisted of the following phases: (a) a text "Be ready" on a screen shown for $1 \mathrm{~s}$, (b) a VC (facial expression depending on the condition) with a name and text "Consider your decision" shown for $5 \mathrm{~s}$ (pre-decision period), (c) a prompt "Decide now whether you collaborate" to choose either to collaborate or not by selecting either $\mathrm{Y}$ for yes or $\mathrm{N}$ for no, (d) the outcome (i.e., decisions made and points received by the VC and participant) revealed $1 \mathrm{~s}$ after the participant's decision and shown for $5 \mathrm{~s}$ (post-decision period), and (e) an intertrial interval varying randomly from 5 to $7 \mathrm{~s}$ while the screen was black.

After finishing with all trials, for a manipulation check, the participant rated all facial expressions of both VCs (static images presented on a computer screen). He or she was asked to rate how well each of the following affect terms described the emotional expression of the VC: happy, sad, angry, and pleasantly relaxed. The ratings were performed on 7-point scales, ranging from 1 (not at all) to 7 (very well). Likewise, the participant rated the emotional expression of the VCs on the valence and arousal dimensions using 9-point pictorial scales [56].

The electrodes were then removed, and the participant was debriefed, given the movie tickets, and thanked for participation.

\subsection{Physiological Data Collection}

The physiological signals were recorded using the Varioport-ARM physiological data acquisition system with 16-bit A/D converters (Becker Meditec, Karlsruhe, Germany). Electrodes mounted in a stretch-Lycra cap (Electrocap; Electro-Cap International, Eaton, $\mathrm{OH}$ ) were used to record EEG activity from left and right frontal (F3, F4), central (C3, C4), and parietal (P3, P4) scalp sites (1020 International System [57]). The electrodes were referred to linked mastoids, and the ground lead was located at the left collarbone (e.g. [48]) Electrode impedances were reduced to less than $5 \mathrm{k} \Omega$. The EEG signal was amplified, band pass-filtered at $0.9-70 \mathrm{~Hz}$, and sampled at a rate of $256 \mathrm{~Hz}$. To facilitate artifact detection, the electrooculogram (EOG) was also measured bipolarly. For vertical eye-movements, the electrodes were placed below and above the right eye; for horizontal eye-movements, the electrodes were placed at the outer canthi of both eyes.

Facial EMG activity was recorded from the left corrugator supercilii, zygomaticus major, and orbicularis oculi muscle regions [58] using surface $\mathrm{Ag} / \mathrm{AgCl}$ electrodes with a contact area of $4 \mathrm{~mm}$ diameter (Becker Meditec). Electrodes were filled with TD-240 electrode gel (Med Assoc. Inc., St. Albans, VT). The raw EMG signal was amplified, band pass-filtered at $57-390 \mathrm{~Hz}$, and sampled at a rate of $1024 \mathrm{~Hz}$.

To enable the responsive VC facial expressions (see above), EDA was recorded with the Varioport digital skin conductance amplifier that applied a constant $0.5 \mathrm{~V}$ across $\mathrm{Ag} / \mathrm{AgCl}$ electrodes with a contact area of $8 \mathrm{~mm}$ diameter (Becker Meditec). Electrodes were filled with TD-246 skin conductance electrode paste (Med Assoc. Inc.) and attached to the middle phalanges of the first and second fingers of the subject's nondominant hand after the hands were washed with soap and water. The EDA signal was sampled at a rate of $32 \mathrm{~Hz}$.

The physiological data were stored on a memory card for offline analysis. In addition, the data were transmitted at a rate of $250 \mathrm{~Hz}$ to the Python script for the real-time emotion assessment. 


\subsection{Data Reduction and Analysis}

EEG data were processed off-line using BrainVision Analyzer 2 (Brain Products, Gilching, Germany). The data were filtered with $0.1-\mathrm{Hz}$ high-pass and $100-\mathrm{Hz}$ low-pass filters, and a 50-Hz notch filter was also applied. Eyemovement artifacts were removed using an ocular correction algorithm [58]. For each trial, the EEG data were segmented into the following epochs: (a) two seconds preceding the onset of the "Be ready" text (baseline), (b) four seconds preceding the participant's decision (predecision period), and (c) three seconds beginning from the second second after the outcome was revealed (postdecision period; for another example of short stimulus periods, i.e., 3-s affective picture viewing, in alpha asymmetry research, see [60]). For these epochs, the power spectra were derived by the fast Fourier transform (FFT) method with a Hanning window (applied to the distal $10 \%$ at each end of the epoch). Power values (in $\mu \mathrm{V}^{2}$ ) within the broad alpha (8-12 Hz [61]) frequency range were extracted for each epoch (in alpha asymmetry research, the $8-13 \mathrm{~Hz}$ frequency band has also been used [62]). Given the previous evidence for differential validity of the lower and upper alpha bands [63], [64], [65], power values were also separately extracted for alpha-1 (8-10 $\mathrm{Hz})$ and alpha-2 (10-12 Hz) bands. As in previous research [62], a frontal asymmetry index (natural log of alpha power on the right minus natural log of alpha power on the left) was computed for each period, using midfrontal sites (F3, F4). Since cortical alpha power is inversely related to cortical activity [66], [67], higher scores on the index indicate greater relative left hemisphere activity.

The facial EMG data were preprocessed using ANSLAB software (version 2.4, University of Basel, Germany). The EMG data were filtered with a $50-\mathrm{Hz}$ notch filter and a $20-\mathrm{Hz}$ high-pass filter, rectified, and smoothed with a 50 -ms moving average window.

For each trial, mean values for facial EMG were derived for (a) baseline (two seconds preceding the onset of the "Be ready" text), (b) pre-decision period (four seconds preceding the participant's decision), and (c) postdecision period (five seconds beginning from the second second after the outcome was revealed). Logarithmic transformations were conducted for facial EMG data to normalize the distributions.

Pre-decision and post-decision physiological data (and emotional ratings for the VC facial expressions) were analyzed by the Linear Mixed-Models (LMM) procedure in SPSS with restricted maximum likelihood estimation. Mixed models allow the use of time-varying predictors (e.g., pre-trial baseline physiological value) and involve a model for the error variance [68]. However, no wellestablished effect size indices are available. Participant ID was specified as the subject variable and trial number was specified as the repeated variable, and AR(1) was specified (on the basis of Schwarz's Bayesian Criterion, BIC) as the covariance structure for the residuals.

When analyzing pre-decision physiological data, participant gender, VC, participant decision at Trial n-1, VC decision at Trial n-1, VC facial expression, pre-trial baseline physiological value, Participant Gender $\times$ VC interac- tion, Participant Decision (at Trial n-1) $\times$ VC decision (at Trial n-1) interaction, and Participant Decision (at Trial n1) $\times$ VC Facial Expression (at Trial n) interaction were specified as fixed effects.

When analyzing post-decision physiological data, participant gender, VC, participant decision at Trial n-1, VC decision at Trial n-1, participant decision at Trial n, VC decision at Trial n, VC facial expression, pre-trial baseline physiological value, pre-decision physiological value, Participant Gender $\times$ VC interaction, Participant Decision (at Trial n-1) $\times$ VC decision (at Trial n-1) interaction, and Participant Decision (at Trial $n$ ) $\times$ VC decision (at Trial $n$ ) interaction were specified as fixed effects.

Participants were treated as a random effect and a random intercept was estimated. The hypotheses were tested with planned contrasts.

When predicting cooperation/defection, the data were analyzed using the Generalized Estimating Equations (GEE) procedure in SPSS. In the GEE procedure, the dependent variable is linearly related to the factors and covariates via a specified link function [69], [70]. The model allows for the dependent variable to have a non-normal distribution and covers widely used statistical models (e.g., logistic models for binary data). The GEE procedure extends the generalized linear model to allow for analysis of repeated measurements or other correlated observations. The GEE approach requires the specification of the correlation structure of the repeated observations of the dependent variable, distribution of the dependent variable, and link function.

We specified participant ID as the subject variable, trial number as the within-subject variable, and a binomial distribution with logistic link for the response variable (decision to cooperate or defect). On the basis of the Corrected Quasi-likelihood under Independence Model Criterion (QICC), we specified independent as the structure of the working correlation matrix. Participant gender, VC, Participant decision at Trial n-1, VC decision at Trial n-1, VC facial expression, baseline and pre-decision physiological variables, and Participant Decision (at Trial $n-1$ ) $\times$

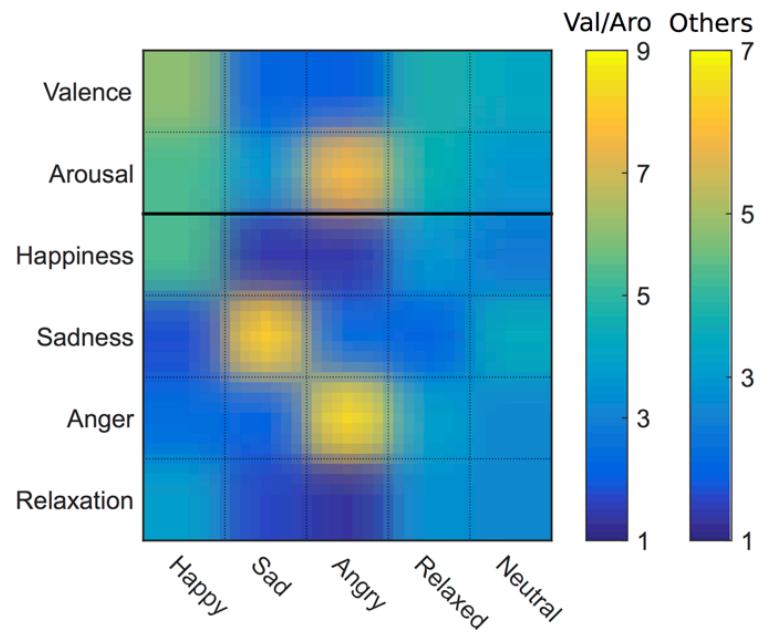

Fig. 2. Confusion matrix for the emotional ratings (rows) for VC facial expressions (columns). Color bars on the right illustrate the used color coding for valence and arousal (recorded on a 1-9 scale) and other ratings (recorded on a 1-7 scale). 
VC Decision (at Trial n-1), Participant Decision (at Trial n1) $\times$ VC Facial Expression, and VC Decision (at Trial n-1) $\times$ VC Facial Expression interactions were included in the GEE model. Bonferroni correction was applied when comparing the levels of the VC facial expression factor (pairwise comparisons).

\section{Results}

\subsection{Manipulation Check}

Figure 2 shows the confusion matrix for the emotional ratings for VC facial expressions (presented after the main experiment). As intended, happy and relaxed facial expressions were rated as showing highest pleasure (most positive emotional valence), whereas sad and angry facial expressions were rated as showing lowest pleasure. In addition, happy and angry facial expressions elicited the highest arousal ratings, whereas sad facial expressions elicited the lowest arousal ratings. However, relaxed facial expressions were rated as showing higher arousal compared to neutral facial expressions. As also intended, happy, sad, and angry facial expressions elicited highest ratings of happiness, sadness, and anger, respectively. However, happy facial expressions tended to elicit higher relaxation ratings compared to (pleasantly) relaxed facial expressions. Thus, in general, the VC facial expressions were rated as showing the intended emotions, with the exception of the relaxed facial expressions that elicited higher arousal ratings than intended. There were, however, also some differences between the two VCs. In general, the male $\mathrm{VC}$ was rated as expressing higher anger compared to the female VC, $F(1,324)=9.56, p=.002$. Sad and angry facial expressions of the male VC tended to be rated as sadder compared to the sad and angry facial expressions of the female VC, whereas happy and neutral facial expressions of the female VC tended to be rated as sadder compared to the happy and neutral expressions of the male VC, $F(4,324)=3.27, p=.012$.

\subsection{Pre-Decision Physiological Responses to VC Facial Expressions}

$\mathrm{H} 2$ predicted that happy and (pleasantly) relaxed VC faces would elicit greater zygomaticus major and orbicularis oculi, and lower corrugator supercilii, facial EMG activity compared to a neutral face, whereas angry and sad VC faces would elicit lower zygomatic and orbicularis oculi activity, and higher corrugator activity, compared to the neutral face. Table 1 shows the mean values for the physiological parameters by condition, and Table 2 shows the results of the contrast analyses. As predicted, the happy and relaxed vs. neutral contrast was significant for all EMG variables, all $p s<.001$, happy and relaxed faces eliciting higher zygomatic and orbicularis oculi, and lower corrugator, activity compared to the neutral face (this was the case particularly for the happy face). As also expected, angry and sad faces elicited higher corrugator activity compared to the neutral face, for the angry and sad vs. neutral contrast, $p<.001$. Consequently, the happy and relaxed vs. angry and sad contrast was also significant for corrugator EMG activity, $p<.001$. However, contrary to our expectation, an angry (but not sad) face elicited also higher zygomatic and orbicularis oculi activity compared to the neutral face, for the angry and sad vs. neutral contrast, $p \mathrm{~s}=.004$ and $<.001$, respectively. The happy and relaxed vs. angry and sad contrast was significant for zygomatic and orbicularis oculi activity, $p \mathrm{~s}=.002$ and .007 , respectively, but this was completely due to greater zygomatic and orbicularis oculi activity in the happy-face condition compared to the sad-face condition.

$\mathrm{H} 3$ predicted that an angry VC facial expression would elicit less zygomatic, orbicularis oculi, and corrugator EMG activity when the participant had defected compared to cooperated in the previous trial. The Participant Cooperates vs. Defects (Previous Trial) $\times$ Angry Face vs. Neutral Face interaction contrast was significant for zygomatic $p=.009$, orbicularis oculi, $p=.001$, and corrugator activity, $p<.001$. Figure 3 shows that, as predicted, an angry (but not neutral) VC evoked lower zygomatic, orbicularis oculi, and corrugator activity when the participant had defected compared to cooperated in the previous trial. It was also found that, when the participant had defected compared to cooperated at Trial n-1, zygomatic activity was higher, $M s=0.19 \ln (\mu \mathrm{V})$ and $0.15 \ln (\mu \mathrm{V})$, respectively, $p=.003$, and corrugator activity was lower, Ms $=0.44 \ln (\mu \mathrm{V})$ and $0.46 \ln (\mu \mathrm{V})$, respectively, $p=.020$, during the pre-decision period of Trial $n$.

$\mathrm{H} 5$ predicted that an angry VC face would elicit diminished relative left frontal activation. All other contrasts were nonsignificant for frontal asymmetry, but an angry $\mathrm{VC}$ face elicited less relative left frontal activation in the

TABLE 1

Means for Pre-Decision Physiological Measures and CoOperation by VC Facial Expression

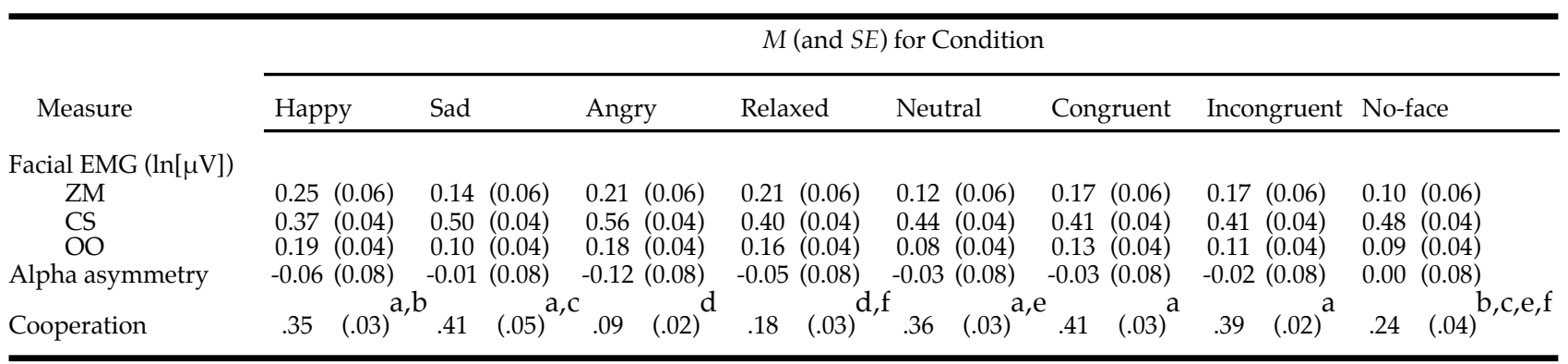

$Z M=$ zygomaticus major; $C S=$ corrugator supercilii; $O O=$ orbicularis oculi. Cooperation = 1 (response), defection =0 (reference category). Mean values with the same superscript are not statistically different (Bonferroni corrected). 
broad alpha frequency band compared to the neutral face, for the angry vs. neutral contrast, $p<.001$. Apparently, this reflected diminished approach motivation during the angry-face condition, given that alpha power at F3 (left site) was higher (i.e., lower cortical activity) during the angry-face compared to neutral condition, $p<.05$, but there was no difference in alpha power at F4 (right site). The difference in frontal asymmetry between the conditions was present also when the analyses were performed separately for the alpha- 1 and alpha- 2 bands, $p s<.01$ and .001 , respectively.

As predicted by $\mathrm{H6}$, an angry (but not neutral) VC evoked less relative left frontal activation in the broad alpha frequency band when the participant had cooperated compared to defected in the previous trial, $p<.001$ for the Participant Cooperates vs. Defects (Previous Trial) $\times$ Angry Face vs. Neutral Face interaction contrast.

In regard to the $R Q$, zygomatic, $p=.016$, and orbicularis oculi, $p=.014$, activity was higher (and corrugator activity tended to be lower) in the congruent expression and incongruent expression conditions compared to the neutral-face condition. However, the congruent expression and incongruent expression conditions did not elicit higher zygomatic and orbicularis oculi activity than the happy-face and relaxed-face conditions. There were no significant differences in facial EMG activity between the congruent expression and incongruent expression conditions, $t \mathrm{~s}<1.2$.
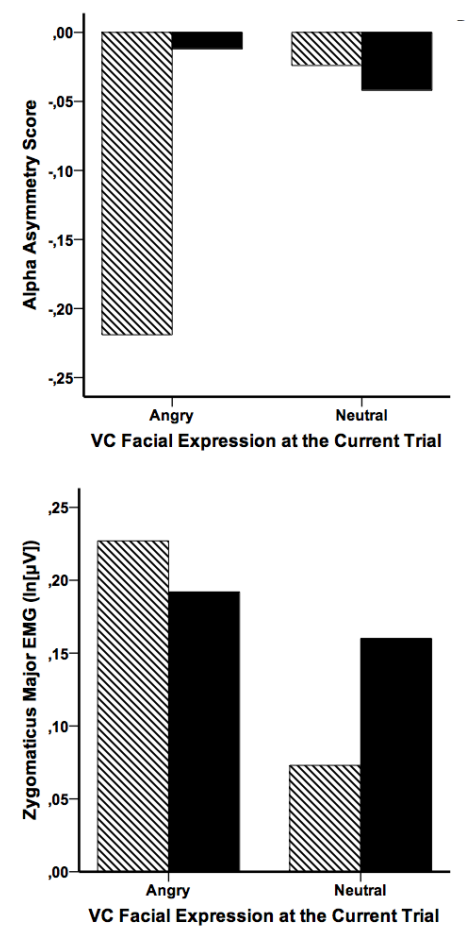

TABLE 2

Summary of Contrast ANALYSES: PRE-DeCISION PhysioLOGICAL RESPONSES TO VC FACIAL EXPRESSIONS

\begin{tabular}{|c|c|c|c|c|}
\hline \multirow[t]{2}{*}{ Variable } & $\begin{array}{l}\text { Contrast } \\
\text { Estimate }\end{array}$ & $S E$ & $d f$ & $t$ \\
\hline & \multicolumn{4}{|c|}{ Happy and relaxed vs. neutral } \\
\hline ZM EMG & 0.22 & 0.04 & 4074.71 & $5.46^{\star * *}$ \\
\hline OO EMG & 0.19 & 0.03 & 4435.60 & $5.96^{* * *}$ \\
\hline \multirow[t]{2}{*}{ CS EMG } & -0.11 & 0.03 & 5137.36 & $-3.47^{* * *}$ \\
\hline & \multicolumn{4}{|c|}{ Angry and sad vs. neutral } \\
\hline ZM EMG & 0.12 & 0.04 & 4094.57 & $2.88^{\star *}$ \\
\hline OO EMG & 0.12 & 0.03 & 4455.75 & $3.77^{\star * *}$ \\
\hline \multirow[t]{2}{*}{ CS EMG } & 0.17 & 0.03 & 5146.45 & $5.23^{* * *}$ \\
\hline & \multicolumn{4}{|c|}{ Angry vs. neutral } \\
\hline Alpha asymmetry & 0.08 & 0.02 & 5182.45 & $3.74^{\star * *}$ \\
\hline Alpha power at F3 & -0.07 & 0.03 & 4654.52 & $-2.20^{*}$ \\
\hline \multirow[t]{2}{*}{ Alpha power at F4 } & -0.01 & 0.03 & 4553.94 & -0.21 \\
\hline & \multicolumn{4}{|c|}{ Happy and relaxed vs. angry and sad } \\
\hline ZM EMG & 0.11 & 0.03 & 4053.21 & $3.17^{* *}$ \\
\hline OO EMG & 0.07 & 0.03 & 4401.78 & $2.71^{* *}$ \\
\hline \multirow[t]{2}{*}{ CS EMG } & -0.28 & 0.03 & 5114.92 & $-10.56^{* * *}$ \\
\hline & \multicolumn{4}{|c|}{ Congruent and incongruent vs. neutral } \\
\hline ZM EMG & 0.10 & 0.04 & 4144.71 & $2.41^{*}$ \\
\hline OO EMG & 0.08 & 0.03 & 4521.63 & $2.47^{*}$ \\
\hline CS EMG & -0.06 & 0.03 & 5182.97 & $-1.92^{\mathrm{a}}$ \\
\hline \multicolumn{5}{|c|}{$\begin{array}{l}\text { Participant Cooperates vs. Defects (at previous trial) } \\
\times \text { Angry Face vs. Neutral Face }\end{array}$} \\
\hline ZM EMG & 0.12 & 0.05 & 4030.90 & $2.60^{* *}$ \\
\hline OO EMG & 0.12 & 0.04 & 4374.18 & $3.24^{* *}$ \\
\hline CS EMG & 0.17 & 0.04 & 5095.83 & $4.47^{* * *}$ \\
\hline Alpha asymmetry & -0.23 & 0.04 & 5165.98 & $-5.08^{* * *}$ \\
\hline \multicolumn{5}{|c|}{ Participant cooperates vs. defects (at previous trial) } \\
\hline ZM EMG & 0.04 & 0.01 & 4292.90 & $3.01^{* *}$ \\
\hline CS EMG & -0.02 & 0.01 & 5244.94 & $-2.32^{*}$ \\
\hline
\end{tabular}

$C S=$ corrugator supercilii; $O O=$ orbicularis oculi; $Z M=$ zygomaticus major . ${ }^{a} p=.055 .{ }^{*} p<.05 .{ }^{* *} p<.01 .{ }^{* * *} p<.001$.
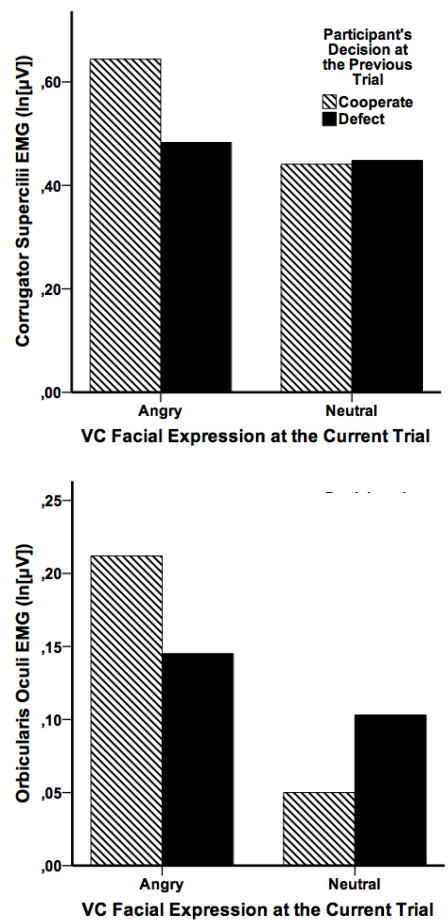

Fig. 3. Pre-decision EEG alpha asymmetry (top left panel) and corrugator supercilii (brow muscle; top right panel), zygomaticus major (cheek muscle; bottom left panel), and orbicularis oculi (periocular muscle; bottom right panel) EMG activity for virtual character (VC) angry and neutral facial expression at the current trial (Trial $n$ ) as a function of participant cooperation and defection at the previous trial (Trial $n-1)$. 
TABLE 3

RESULtS OF GEE ANALYSIS OF DECISION DATA

\begin{tabular}{lrrr}
\hline \multicolumn{1}{c}{ Variable } & Wald $\chi^{2}$ & $\mathrm{df}$ & \multicolumn{1}{c}{$p$} \\
\hline (Intercept) & 48.424 & 1 & $<.001$ \\
Participant gender & 0.122 & 1 & .727 \\
VC & 4.436 & 1 & .035 \\
Participant decision at Trial n-1 & 17.939 & 1 & $<.001$ \\
VC decision at Trial n-1 & 3.884 & 1 & .049 \\
VC facial expression & 95.706 & 7 & $<.001$ \\
Participant decision at Trial n-1 & & & \\
$\quad$ × VC decision at Trial n-1 & 2.491 & 1 & .114 \\
Participant decision at Trial n-1 & & & \\
$\quad$ × VC facial expression & 2.359 & 7 & .937 \\
VC decision at Trial n-1 & & & \\
$\quad \times$ VC facial expression & 13.051 & 7 & .071 \\
Alpha asymmetry (baseline) & 8.807 & 1 & .003 \\
Alpha asymmetry (pre-decision) & 34.439 & 1 & $<.001$ \\
ZM EMG (baseline) & 0.005 & 1 & .949 \\
ZM EMG (pre-decision) & 0.063 & 1 & .802 \\
CS EMG (baseline) & 3.045 & 1 & .081 \\
CS EMG (pre-decision) & 10.308 & 1 & .001 \\
OO EMG (baseline) & 1.729 & 1 & .188 \\
OO EMG (pre-decision) & 0.054 & 1 & .816 \\
\end{tabular}

For decision, cooperation $=1$ (response), defection $=0$ (reference catego$r y)$.

\subsection{Predictors of Cooperation/Defection}

$\mathrm{H} 1$ predicted that seeing a happy VC facial expression during the pre-decision period would elicit cooperation, especially when the VC had collaborated in the previous trial, whereas an angry VC facial expression would elicit defection. Table 1 shows collaboration by condition, and Table 3 shows the results of the GEE analysis of decision data. The happy-face condition didn't differ from the neutral and no-face conditions, and the interaction of VC decision at Trial n-1 with VC facial expression was nonsignificant. However, as hypothesized, an angry VC facial expression predicted less frequent collaboration compared to the other conditions (Bonferroni-corrected $p \mathrm{~s}<$ .001 , but for the no-face condition, $p=.032$ ), with the ex-

TABLE 4

Summary of Contrast ANALYSES: Post-DeCISION PhySIOLOGICAL RESPONSES

\begin{tabular}{|c|c|c|c|c|}
\hline Variable & $\begin{array}{l}\text { Contrast } \\
\text { Estimate }\end{array}$ & $S E$ & $d f$ & $t$ \\
\hline & \multicolumn{4}{|c|}{ Participant defects vs. cooperates (at Trial $n$ ) } \\
\hline OO EMG & -0.05 & 0.01 & 5286.41 & $-5.83^{* * *}$ \\
\hline ZM EMG & -0.06 & 0.01 & 5288.83 & $-6.00^{* * *}$ \\
\hline \multirow[t]{2}{*}{ CS EMG } & -0.03 & 0.01 & 5277.51 & $-3.31^{* * *}$ \\
\hline & \multicolumn{4}{|c|}{ VC defects vs. cooperates (at Trial $\mathrm{n}$ ) } \\
\hline Alpha asymmetry & -0.03 & 0.01 & 5188.36 & $-2.47^{*}$ \\
\hline Alpha-2 asymmetry & -0.06 & 0.02 & 5251.39 & $-3.13^{* *}$ \\
\hline OO EMG & 0.06 & 0.01 & 5264.88 & $7.39^{* * *}$ \\
\hline \multirow[t]{3}{*}{ ZM EMG } & 0.06 & 0.01 & 5265.25 & $5.81^{* * *}$ \\
\hline & \multirow{2}{*}{\multicolumn{4}{|c|}{ Participant Defects vs. Cooperates (at Trial n) }} \\
\hline & & & & \\
\hline OO EMG & 0.06 & 0.02 & 5266.05 & $4.18^{\star \star \star}$ \\
\hline ZM EMG & 0.08 & 0.02 & 5266.87 & $4.13^{\star * *}$ \\
\hline \multirow[t]{2}{*}{ CS EMG } & 0.03 & 0.01 & 5260.05 & $2.31^{*}$ \\
\hline & $\begin{array}{l}\text { ipant Defe } \\
\text { C Defects }\end{array}$ & $\begin{array}{ll}\text { cts vs } \\
\text { vs. Co }\end{array}$ & $\begin{array}{l}\text { ooperates } \\
\text { erates (at }\end{array}$ & $\begin{array}{l}\text { at Trial n-1) } \\
\text { rial } n-1 \text { ) }\end{array}$ \\
\hline Alpha asymmetry & -0.06 & 0.03 & 5188.92 & $-2.42^{*}$ \\
\hline Alpha-2 asymmetry & -0.09 & 0.04 & 5252.01 & $-2.54^{*}$ \\
\hline OO EMG & -0.05 & 0.02 & 5265.96 & $-3.11^{* *}$ \\
\hline ZM EMG & -0.05 & 0.02 & 5266.87 & $-2.52^{*}$ \\
\hline
\end{tabular}

${ }^{*} p<.05 .{ }^{* *} p<.01 .{ }^{* * *} p<.001$ ception of the relaxed VC faces, $p=.859$. Happy and sad VC faces did not differ from neutral faces and the no-face condition, $p s>.23$. The congruent expression and incongruent expression conditions were the only that elicited significantly more collaboration than the no-face condition, $p s=.023$ and .003 , respectively, but they did not differ from the neutral-face condition, $p s>0.90$.

H4 predicted that high corrugator supercilii EMG activity during the pre-decision period would predict defection, whereas high zygomatic and orbicularis oculi activity would predict cooperation. As expected, high corrugator activity was associated with defection, $B=-.685, p=$ .001. Zygomatic and orbicularis oculi activity was not associated with collaboration/defection.

As suggested by $\mathrm{H7}$, relatively greater left frontal activation predicted defection, $B=-.467, p<.001$. Alpha power at F4 (right site) was not associated with defection/ collaboration, $B=-.023, p=.806$, and the association of low alpha power (high cortical activity) at F3 (left site) with defection narrowly failed to reach statistical significance, $B=.152, p=.062$.
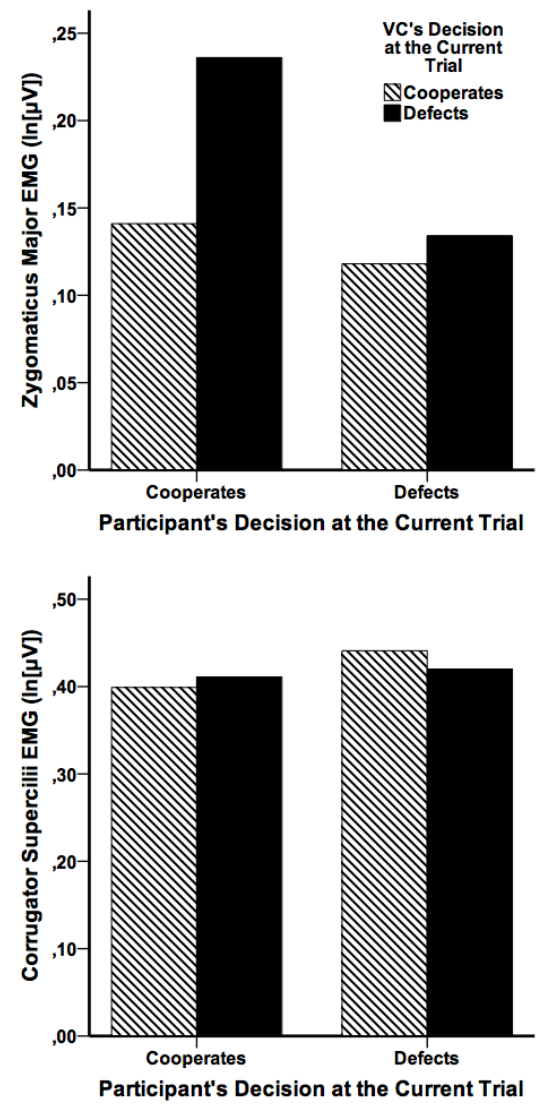

Fig. 4. Post-decision zygomaticus major (cheek muscle; top panel) and corrugator supercilii (brow muscle; bottom panel) EMG activity for participant cooperation and defection at the current trial (Trial $n$ ) as a function of virtual character (VC) cooperation and defection at the current trial. 

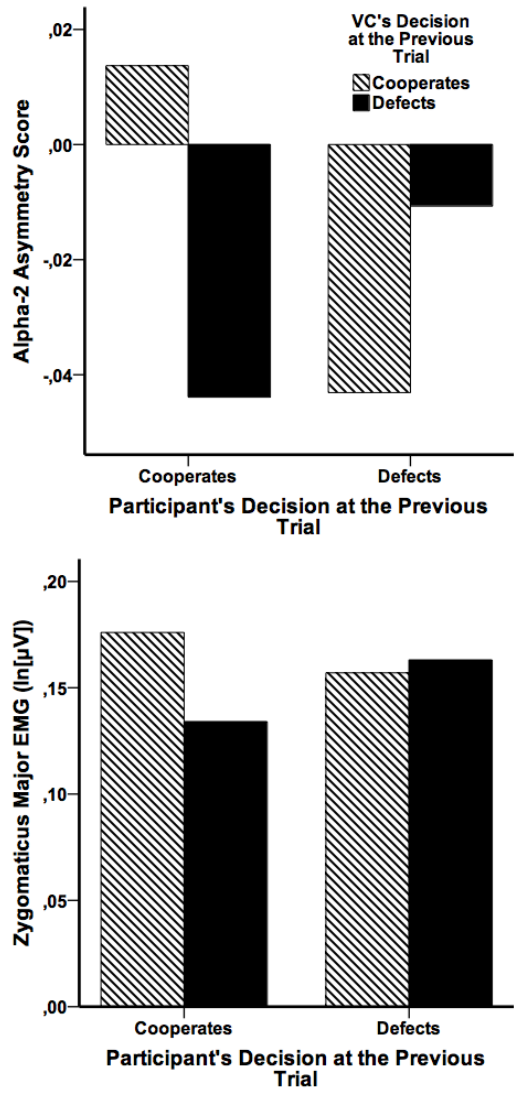

Fig. 5. Post-decision alpha-2 asymmetry (top panel) and zygomatic EMG activity (cheek muscle; bottom panel) at the current trial (Trial $n$ ) for participant cooperation and defection at the previous trial (Trial n-1) as a function of virtual character (VC) cooperation/defection at the previous trial.

\subsection{Post-Decision Physiological Responses}

H8 suggested that zygomatic and orbicularis oculi EMG activity would increase, and corrugator activity would decrease, as a function of payoffs. Table 4 shows the results of the contrast analyses. The interaction contrast Participant Defects vs. Cooperates (at Trial n) $\times$ VC Defects vs. Cooperates (at Trial $\mathrm{n}$ ) was significant in predicting zygomatic, orbicularis oculi, and corrugator activity, $p \mathrm{~s}<.001,<.001$, and $=.021$, respectively. Unexpectedly, as shown in Figure 4, zygomatic activity was highest when the participant cooperated and the VC defected (i.e., lowest payoff) and lowest when the participant defected and the VC cooperated (i.e., highest payoff). Importatntly, exactly the same held true also for orbicularis oculi activity. Corrugator activity was highest when the participant defected and the VC cooperated (i.e., highest payoff) and lowest when both the participant and VC cooperated. There were also significant main effects; that is, zygomatic, $M s=0.19 \ln (\mu \mathrm{V})$ and $0.13 \ln (\mu \mathrm{V})$, and orbicularis oculi activity, $M s=0.17 \ln (\mu \mathrm{V})$ and $0.12 \ln (\mu \mathrm{V})$, was higher, and corrugator activity was lower, $M s=0.41 \ln (\mu \mathrm{V})$ and $0.43 \ln (\mu \mathrm{V})$, when the participant had collaborated compared to defected, respectively, all $p s<.001$. In addition, zygomatic, $M s=0.13 \ln (\mu \mathrm{V})$ and $0.19 \ln (\mu \mathrm{V})$, and orbicularis oculi activity, $M s=0.12 \ln (\mu \mathrm{V})$ and $0.18 \ln (\mu \mathrm{V})$, was lower when the VC had collaborated compared to defected, respectively, $p \mathrm{~s}<.001$. However, VC collaboration elicited greater relative left frontal activation compared to VC defection, $M s=-0.01$ and -0.04 , respectively, $p=.014$. This likely reflected increased approach motivation, given that alpha power at F3 (left site) tended to be lower (i.e., higher cortical activity) after VC collaboration compared to defection, $p=.075$, but there was clearly no difference in alpha power at F4 (right site; $p=.866$ ). When analyzed separately, the difference in frontal asymmetry between VC collaboration and defection was significant for the alpha-2 frequency band, $M s=0.01$ and -0.05 , respectively, $p=.002$, but not for the alpha- 1 frequency band, $p=.211$.

Not only the decisions at the current trial (Trial $n$ ) but also at the previous trial (Trial n-1) influenced postdecision frontal asymmetry and EMG responses at Trial n. That is, there was a significant interaction contrast Participant Defects vs. Cooperates (at Trial n-1) $\times$ VC Defects vs. Cooperates (at Trial n-1) in predicting alpha asymmetry and zygomatic and orbicularis oculi activity, $p \mathrm{~s}=$ $.015, .012$, and .002 , respectively. When analyzed separately, the interaction was significant for the alpha-2 frequency band, $p=.011$, but not for the alpha- 1 frequency band, $p=.153$. As shown by Figure 5, relative left frontal activation in the alpha-2 frequency band was greatest when both the participant and VC had collaborated at Trial n-1 and least when the participant and VC made different decisions. Also, both zygomatic and orbicularis oculi activities were highest when both the participant and VC had collaborated at Trial n-1 and lowest when the participant had collaborated, but the VC had defected.

\section{Discussion}

The present investigation is the first to show how VC dynamic facial expressions influence frontal EEG asymmetry (putatively indexing inner emotional-motivational processes) and facial EMG responses (emotional expressions) in the context of social decision making and how the physiological responses predict the human decision to cooperate.

\subsection{Facial Responses to VC Facial Expressions}

In general, VC facial expressions elicited congruent facial muscle activity. That is, happy VC faces elicited increased zygomatic and orbicularis oculi, and decreased corrugator, EMG activity, whereas angry and sad VC faces elicited increased corrugator activity. This is in contrast to previous studies that have failed to find increased corrugator activity in response to an angry VC expression [30], [35]. However, unexpectedly, we also found that angry VC faces elicited increased zygomatic and orbicularis oculi EMG activity (this may relate to the effect of preceding interactional events).

Importantly, the results supported our hypothesis that human facial expressions elicited by VC emotional expressions would depend on the previous interactional events and be affected by display rules (see [15]). An angry VC facial expression elicited less zygomatic, orbicularis oculi, and corrugator EMG activity (less smiling and frowning) when the participant had defected compared to cooperated in the previous trial. This is in line with the 
view that people may avoid smiling (smiling may be interpreted as malicious pleasure) when another person shows anger in response to your defection. Another person's apparently justifiable anger expression is also expected to elicit less frowning compared to unjustifiable anger expression. These findings extend the Computers as Social Actors paradigm (see [52]) and may suggest that the application of display rules is habitual behavior.

\subsection{Facial Expressions Predicting Cooperation}

Although a happy VC facial expression didn't increase collaboration, we found that both an angry VC facial expression and high pre-decision corrugator supercilii EMG activity (related to frowning) predicted human defection. This was expected as an angry and frowning expression would be expected to signal antagonism rather than willingness to engage in cooperative behavior. Anticipated VC defection apparently leads to reciprocal human defection. However, in disagreement with the study by Scharlemann et al. ([25]; who used photographs of smiling faces), we didn't find happy (smiling) VC faces to elicit human cooperation.

\subsection{Inner Emotional-Motivational Processes during Human-VC Interaction}

The results supported our expectation that pre-decision inner emotional-motivational processes and emotional facial expressions would be dissociated and frontal EEG asymmetry would primarily be related to pre-goal attainment emotion (see [50]). That is, an angry VC face appeared not to evoke approach-motivated anger (see [71]), given that it elicited less (not greater) relative left frontal activation compared to the neutral face. Analyses carried out separately by hemisphere also suggested that this reflected diminished approach motivation rather than increased withdrawal motivation. The results also showed that the decrease in relative left frontal activation elicited by an angry VC was particularly pronounced when the participant had cooperated in the previous trial. This fits to the view that an angry VC face may be perceived as a cue of forthcoming nonreward (VC defection resulting in a failure to achieve the goal of getting a high payoff), which is particularly disappointing after one has cooperated, thereby resulting in decreased approach motivation. Thus, also pre-decision inner emotionalmotivational processes elicited by VC emotional expressions appeared to depend on the previous interactional events, although in a different way than human facial expressions.

As expected, relatively greater left frontal activation during the pre-decision period predicted human defection. This also conforms to the idea that pre-decision frontal asymmetry is linked to the anticipatory ("wanting") phase of goal (i.e., high payoff) attainment rather than to the motivation to establish cooperation per se. That is, given the payoff matrix, human defection was a prerequisite for getting a high payoff. It is of interest, however, that decreased resting right-lateralized activity has been associated with low levels of the implicit affiliation motive [72]. Likewise, Schweiger, Stemmler,
Burgdorf, and Wacker [73] found that a decrease in relative left-frontal cortical activity was associated with an increase in the emotion warmth-liking (related to feelings of affection and acceptance) and interpersonal trust in a trust game. However, they argued that these affiliative processes are linked to the consummatory ("liking") phase of attaining an affiliative goal, thereby exerting a right-lateralizing effect.

\subsection{Post-Decision Facial Expressions and Frontal Asymmetry}

We predicted that post-decision positive facial expressions will increase (and negative facial expressions will decrease) as a function of payoffs. However, we found an opposite relationship - that is, both zygomatic and orbicularis oculi EMG activity (associated with smiling) were highest when the payoff was lowest (human cooperated and VC defected, i.e., a human failure), and lowest when the payoff was highest (human defected and VC cooperated). Corrugator activity (associated with frowning) was also highest when the payoff was highest. Interestingly, several previous studies have shown that the death of the player's own character in digital (e.g., first-person shooter) games elicits activation of the zygomaticus major and orbicularis oculi (smile after dying) and deactivation of the corrugator supercilii [74], [75], [76]. Such events as player death or getting the lowest payoff in the Prisoner's Dilemma game clearly reflect defeat in the light of the player's goal. Thus, the present study supports the view that, when failing to achieve one's goal, people may sometimes express (by smiling) the opposite emotion of what they are actually feeling (potentially for the purposes of emotional coping). The dissociation of emotional expressions and inner emotional-motivational processes is also revealed by the fact that VC collaboration elicited greater relative left frontal activation in the alpha- 2 frequency band than VC defection. Thus, as expected, frontal EEG asymmetry was not associated with post-goal attainment emotion, but to interaction partner cooperation.

Importantly, we found that not only decisions at the current trial of the game but also decisions at the previous trial influenced post-decision physiological responses at the current trial. Both positive facial expressions and relative left frontal activation in the alpha-2 frequency band were greatest when both the participant and VC had cooperated at the trial preceding the current trial. A lack of reciprocity, in turn, resulted in less positive facial expressions and less relative left frontal activation. It appears that people ruminate over the reciprocity of their social interaction, which may have emotional effects manifesting only after some time. Given that reciprocal cooperation at the previous trial appears to be linked to the consummatory phase of attaining an affiliative goal, the present finding may be at variance with the suggestion that increased right-lateralized activity should be present during this phase [73].

\subsection{Affective Congruency of VC Facial Expressions}

Given the evidence that behavioral mimicry creates rap- 
port (e.g., [77]), our secondary research question asked whether the congruency between VC (pre-decision) emotional expression and the participant's emotional state (as inferred on the basis of facial EMG activity and EDA) influences human cooperation and physiological responses. However, we found no differences between the congruent and incongruent expression conditions. It is of note that the present study provides only a weak test for the effect of affective congruency. Although pre-tests suggested that VC facial expressions were dictated by human emotional/physiological responses as intended, this was not necessarily completely the case for all participants. Also, the present study didn't take into account that the effects of emotion expression congruency on cooperation may vary by the type of emotion (e.g., happy vs. angry expression). It may also be that the use of facial EMG activity (emotional expressions) alone would have been better than a combination of EMG and EDA in determining the VC facial expressions.

\subsection{Limitations}

Evidently, the validity of our results depends on the validity of VC emotional expressions. In general, the VC facial expressions were rated as showing the intended emotions, but the relaxed facial expressions elicited higher arousal ratings than intended. However, this does not affect the tests of our main hypotheses, but may have contributed to the lack of affective congruency findings. Nevertheless, there were clear limitations in the VC facial expressions as they were not exact representations of the target emotions in terms of action units. There were also only two VCs that differed not only in terms of gender (not possible to make conclusions on the effects of $\mathrm{VC}$ gender). Also, the participants may have learned the lack of correlation between VC facial expressions and VC decisions (although the complexity of the design may make this difficult), which could weaken the effects found. Future studies might also involve fewer trials, but longer interactions with the same $\mathrm{VC}$, which may increase involvement in the interaction. In general, more focused (e.g., online) experiments are needed to acquire a deeper understanding of the present phenomena (including affective congruency). An additional limitation was the quite small sample size, but most of the associations found were strong. Also, given the small number of EEG electrodes, it was not possible to use source localization.

\section{Conclusion}

The present study showed that both human pre-decision frontal EEG asymmetry (putatively indexing inner emotional-motivational processes) and facial EMG activity (emotional expressions) elicited by an angry VC facial expression varied as a function of preceding interactional events (human collaboration/defection). However, predecision inner emotional-motivational processes and emotional facial expressions were dissociated, suggesting that human goals influence pre-decision frontal asymmetry, whereas display rules may affect (pre-decision) emotional expressions in human-VC interaction. The study also showed that an angry VC facial expression, high human pre-decision corrugator EMG activity (related to frowning), and relatively greater left frontal activation (linked to the "wanting" phase of goal attainment) predicted the human decision to defect, whereas both post-decision frontal asymmetry and facial EMG activity were related to reciprocal cooperation. The results are in line with the view that the justifiability of VC emotional expressions and the perceived fairness of $\mathrm{VC}$ actions influence human emotional responses. That is, people appear to respond to VCs as they would be expected to respond in human-human interaction. The study provides additional evidence that VCs are a powerful research tool and can be used to study basic social phenomena. The result may also have practical implications when designing game characters, service agents, and persuasive technology, especially when the interaction involves negotiating.

\section{ACKNOWLEDGMENT}

This work was supported by the Academy of Finland, project number 268999.

\section{REFERENCES}

[1] D. Kahneman, I. Ritov and D. Schkade, "Economic preferences or attitude expressions? An analysis of dollar responses to public issues," J. Risk and Uncertainty, vol. 19, pp. 203-235, 1999.

[2] Loewenstein, George, and Jennifer S. Lerner, "The role of affect in decision making," Handb. Affect. Sci., R. J. Davidson, K. R. Scherer, and H. H. Goldsmith, eds., New York: Oxford University Press, pp. 619-642, 2003.

[3] B. Shiv, and A. Fedorikhin, "Heart and mind in conflict: The interplay of affect and cognition in consumer decision making," J. Consum. Res., vol. 26, pp. 278-93, 1999.

[4] P. Slovic, M. L. Finucane, E. Peters and D. G. MacGregor, "Risk as analysis and risk as feelings: Some thoughts about affect, reason, risk, and rationality," Risk Anal., vol. 24, pp. 311-322, 2004.

[5] R. Axelrod and W. D. Hamilton, "The evolution of cooperation," Science, vol. 211, pp. 1390-96, 1981.

[6] G. Bente, O. Baptist and H. Leuschner, "To buy or not to buy: Influence of seller photos and reputation on buyer trust and purchase behavior," Int. J. Human-Comput. Stud., vol. 70, no. 1, pp. 1-13, 2012.

[7] D. Gill, O.B. Garrod, R.E. Jack and P.G. Schyns, "Facial movements strategically camouflage involuntary social signals of face morphology," Psychol. Sci., vol. 25, no.5, pp. 1079-1086, 2014.

[8] M. Mehu, K. Grammer, and R. Dunbar, "Smiles when sharing," Evol. and Human Behav., vol. 28, no. 6, pp. 415-422, 2007.

[9] J. Schug, D. Matsumoto, Y. Horita, T. Yamagishi, and K. Bonnet, "Emotional expressivity as a signal of cooperation," Evol. and Human Behav., vol. 31, no. 2, pp. 87-94, 2010.

[10] C. de Melo, P. Carnevale, and J. Gratch, "The effect of expression of anger and happiness in computer agents on negotiations with humans," The 10th Int. Conf. on Autonomous Agents and Multiagent Systems-Vol. 3, K. Tumer, P. Yolum, L. Sonenberg, \& P. Stone, eds., Richland, SC: International Foundation for Autonomous Agents and Multiagent Systems, pp. 937-944, 2011.

[11] C. de Melo, P. J. Carnevale, and J. Gratch. "The impact of emo- 
tion displays in embodied agents on emergence of cooperation with people," Presence, vol. 20, no. 5, pp. 449-465, 2011.

[12] C. de Melo, J. Gratch and P. Carnevale, "Humans vs. computers: Impact of emotion expressions on people's decision making," IEEE Trans. Affect. Comput., vol. 6, pp.127-136, 2015

[13] G. Bente, N. C. Krämer and F. Eschenburg, "Is there anybody out there? Analyzing the effects of embodiment and nonverbal behavior in avatar-mediated communication", Mediated Interpersonal Commun., E. Konijn, S. Utz, M. Tanis and S. Barnes, eds., Mahwah, NJ: Lawrence Erlbaum Associates, pp. 131-157, 2008.

[14] A. Choi, C. de Melo, W. Woo, and J. Gratch, "Affective engagement to emotional facial expressions of embodied social agents in a decision-making game," Comput. Animat. Virtual Worlds, vol. 23, no. 3-4, pp. 331-342, 2014.

[15] R. Zaalberg, A. Manstead, and A. Fischer, "Relations between emotions, display rules, social motives, and facial behavior," Cogn. Emotion, vol. 18, no. 2, pp. 183-207, 2004.

[16] P. Ekman, "Strong Evidence for universals in facial expressions," Psychol. Bull., vol. 115, no. 2, pp. 268-287, 1994.

[17] A. Fridlund, "Sociality of solitary smiling: Potentiation by an implicit audience," J. Personal. Soc. Psychol., vol. 60, no. 2, pp. 229-240, 1991.

[18] U. Hess, R. Banse, and A. Kappas. "The intensity of facial expression is determined by underlying affective state and social situation," J. Pers. Soc. Psychol., vol. 69, no. 2 pp. 280-288, 1995.

[19] E. Jakobs, A. Manstead, and A. Fischer, "Social motives and emotional feelings as determinants of facial displays: The case of smiling," Pers. Soc. Psychol. Bull., vol. 25, no. 4 pp. 424-435, 1999.

[20] B. Parkinson, "Do facial movements express emotions or communicate motives?," Pers. Soc. Psychol. Rev., vol. 9, no. 4, pp. 278-311, 2005.

[21] K. Scherer, "The nature and dynamics of relevance and valence appraisals: Theoretical advances and recent evidence," Emotion Rev., vol. 5, no. 2, pp. 150-162, 2013.

[22] D. De Cremer, "Trust and fear of exploitation in a public goods dilemma," Curr. Psychol., vol. 18, no. 2, pp. 153-163, 1999.

[23] E. Krumhuber, A. Manstead, D. Cosker, D. Marshall, P. Rosin, and A. Kappas. "Facial dynamics as indicators of trustworthiness and cooperative behavior," Emotion, vol. 7, no. 4, pp. 730735, 2007, doi:10.1037/1528-3542.7.4.730.

[24] W. Ross and J. LaCroix, "Multiple meanings of trust in negotiation theory and research: A literature review and integrative model," Int. J. Conflict Management, vol. 7, pp. 314-360, 1996.

[25] J. Scharlemann, C. Eckel, A. Kacelnik, and R. Wilson, "The value of a smile: Game theory with a human face," J. Econ. Psychol., vol. 22, no. 5, pp. 617-640, 2001.

[26] D. Keltner and A. Kring, "Emotion, social function, and psychopathology," Rev. Gen. Psychol., vol. 2, pp. 320-342, 1998.

[27] M. Morris and D. Keltner, "How emotions work: The social functions of emotional expression in negotiations," Res. Organ. Behav., vol. 2, pp. 1-50, 2000.

[28] C. de Melo, L. Zheng and J. Gratch, "Expression of moral emotions in cooperating agents," Intelligent Virtual Agents, Z. Ruttkay, M. Kipp, A. Nijholt and H.H. Vilhjálmsson, eds., Heidelberg: Springer-Verlag, pp. 301-307, 2009.

[29] R. Frank, "Introducing Moral Emotions into Models of Rational Choice," Feelings and Emotions, A. Manstead, N. Frijda and A. Fischer, eds., Cambridge University Press, pp. 422-440, 2004.
[30] F. Schrammel, S. Pannasch, S. Graupner, A. Mojzisch, and B. Velichkovsky, "Virtual friend or threat? The effects of facial expression and gaze interaction on psychophysiological responses and emotional experience," Psychophysiol., vol. 46, no. 5, pp. 922-931, 2009.

[31] U. Dimberg, "Psychophysiological reactions to facial expressions," Nonverbal Commun., U. Segerstråle and P. Molnar, eds., Mahwah, NJ: Erlbaum. pp. 47-60, 1997.

[32] U. Dimberg and M. Petterson, "Facial reactions to happy and angry facial expressions: Evidence for right hemisphere dominance," Psychophysiol., vol. 37, no. 5, pp. 693-696, 2000.

[33] V. Surakka and J. Hietanen, "Facial and emotional reactions to Duchenne and non-Duchenne smiles," Int. J. Psychophysiol., vol. 29, no. 1, pp. 23-33, 1998.

[34] U. Hess, P. Philippot and S. Blairy, "Facial reactions to emotional facial expressions: affect or cognition?," Cogn. Emotion, vol. 12, no. 4, pp. 509-531, 1998.

[35] P. Weyers, A. Mühlberger, C. Hefele and P. Pauli, "Electromyographic responses to static and dynamic avatar emotional facial expressions," Psychophysiol., vol. 43, no. 5, pp. 450-453, 2006.

[36] N. Ravaja, "Contributions of psychophysiology to media research: Review and recommendations," Media Psychol., vol. 6, no. 2, pp. 193-235, 2004.

[37] P. Ekman, Paul and W. Friesen, Unmasking The Face, Cambridge, MA: Malor Books, 2003

[38] J. Cacioppo and. W. Gardner, "Emotion," Annu. Rev. Psychol., vol. 50, no. 1, pp. 191-214, 1999.

[39] M. Pillutla and J. Murnighan, "Unfairness, anger, and spite: Emotional rejections of ultimatum offers," Organ. Behav. Human Decision Processes, vol. 68, no. 3, pp. 208-224, 1996.

[40] R. Davidson, "Affective neuroscience and psychophysiology: toward a synthesis," Psychophysiol., vol. 40, pp. 655-665, 2003.

[41] R. Davidson, "What does the prefrontal cortex "do" in affect: perspectives on frontal EEG asymmetry research," Biol. psychol., vol. 67, no. 1, pp. 219-234, 2004.

[42] J. Coan and J. Allen, "Frontal EEG asymmetry as a moderator and mediator of emotion." Biol. psychol., vol. 67, pp. 7-50, 2004.

[43] H. Demaree, D. Everhart, E. Youngstrom, and D. Harrison, "Brain lateralization of emotional processing: historical roots and a future incorporating "dominance"," Behav. Cogn. Neurosci. Rev., vol. 4, no. 1, pp. 3-20, 2005.

[44] D. Pizzagalli, R. Sherwood, J. Henriques, and R. Davidson, "Frontal brain asymmetry and reward responsiveness a sourcelocalization study," Psychol. Sci., vol. 16, pp. 805-813, 2005.

[45] L. Gianotti, D. Knoch, P. Faber, D. Lehmann, R. Pascual-Marqui, C. Diezi, C. Schoch, C. Eisenegger, and E. Fehr, "Tonic activity level in the right prefrontal cortex predicts individuals' risk taking," Psychol. Sci., vol. 20, no. 1, pp. 33-38, 2009.

[46] D. Knoch, L. Gianotti, T. Baumgartner and E. Fehr, "A neural marker of costly punishment behavior," Psychol. Sci., vol. 21, pp. 337-342, 2010.

[47] E. Hatfield, J. Cacioppo, and R. Rapson, Emotional Contagion, New York: Cambridge university press, 1994.

[48] E. Harmon-Jones, J. Allen and E. Barratt, "Anger and prefrontal brain activity: EEG asymmetry consistent with approach motivation despite negative affective valence," J. Pers. Soc. Psychol. vol. 74, pp. 1310-1316, 1996.

[49] E. Harmon-Jones and J. Sigelman, "State anger and prefrontal brain activity: Evidence that insult-related relative left prefrontal activation is associated with experienced anger and aggres- 
sion," J. Pers. Soc. Psychol., vol.80, pp. 797-803, 2001.

[50] R. Davidson, J. Marshall, A. Tomarken and J. Henriques, "While a phobic waits: Regional brain electrical and autonomic activity in social phobics during anticipation of public speaking," Biol. Psychiatry, vol. 47, no. 2, pp. 85-95, 2000.

[51] D. Watson, D. Wiese, J. Vaidya and A. Tellegen, "The two general activation systems of affect: Structural findings, evolutionary considerations, and psychobiological evidence," J. Pers. Soc. Psychol., vol. 76, no. 5, pp. 820-838, 1999.

[52] C. Nass and K. M. Lee, "Does computer-synthesized speech manifest personality? Experimental tests of recognition, similarity-attraction, and consistency-attraction," J. Exp. Psychol., vol. 7, no. 3, pp. 171-181, 2001.

[53] J. Russell, "A circumplex model of affect," J. Personal. Soc. psychol., vol. 39, no. 6, pp. 1161-1178, 1980

[54] P. Ekman and W. Friesen, Pictures of Facial Affect, Palo Alto, California: Consulting Psychologists Press, 1976.

[55] P. Ekman, "Universal and cultural differences in facial expression of emotion," Nebraska Symposium on Motivation, J. Cole., ed., vol. 19, Lincoln: University of Nebraska Press, pp. 207-284, 1972.

[56] P. Lang, "Behavioral treatment and bio-behavioral assessment: Computer applications", Technol. Mental Health Care Delivery Systems, J. Sidowski, J. Johnson and T. Williams, eds., Norwood, NJ: Ablex, pp. 119-137, 1980.

[57] H. Jasper, "The ten-twenty electrode system of the international federation", Electroencephalography and Clinical Neurophysiol., vol. 10, pp. 371-375, 1958.

[58] L. Tassinary, J. Cacioppo and E. Vanman, "The skeletomotor system: Surface electromyography." Handb. Psychophysiol., vol. 2, pp. 163-199, 2000.

[59] G. Gratton, M. Coles and E. Donchin, "A new method for offline removal of ocular artifact," Electroencephalography and Clinical Neurophysiol., vol. 55, no. 4, pp. 468-484, 1983.

[60] E. Harmon-Jones, Eddie, L. Lueck, M. Fearn and C. HarmonJones, "The effect of personal relevance and approach-related action expectation on relative left frontal cortical activity," Psychol. Sci., vol. 17, no. 5, pp. 434-440, 2006.

[61] G. Buzsáki, Rhythms of the Brain, New York: Oxford University Press, 2006.

[62] J. Allen, J. Coan and M. Nazarian, "Issues and assumptions on the road from raw signals to metrics of frontal EEG asymmetry in emotion," Biol. Psychol., vol. 67, no. 1, pp. 183-218, 2004.

[63] I. Goncharova and R. Davidson, "The factor structure of EEG: Differential validity of low and high alpha power asymmetry in predicting affective style", Psychophysiol., vol. 32/S1, p. S35, 1995.

[64] W. Klimesch, "EEG alpha and theta oscillations reflect cognitive and memory performance: a review and analysis," Brain Res. Rev., vol. 29, no. 2, pp. 169-195, 1999.

[65] I. Papousek and G Schulter, "Covariations of EEG asymmetries and emotional states indicate that activity at frontopolar locations is particularly affected by state factors," Psychophysiology, vol. 39, no. 3, pp. 350-360, 2002.

[66] I. Cook, R. O'Hara, S. Uijtdehaage, M. Mandelkern and A. Leuchter, "Assessing the accuracy of topographic EEG mapping for determining local brain function," Electroenceph. Clin. Neurophysiol., vol. 107, no. 6, pp. 408-414, 1998.

[67] D. Lindsley and J. Wicke, "The electroencephalogram: Autonomous electrical activity in man and animals," Bioelectric Record- ing Techniques, vol. 1 (part B), pp. 3-83, 1974.

[68] E. Bagiella, R. Sloan and D. Heitjan, "Mixed-effects models in psychophysiology," Psychophysiol., vol. 37, pp. 13-20, 2000.

[69] K. Liang and S. Zeger, "Longitudinal data analysis using generalized linear models," Biometrika, vol. 73, no. 1, pp. 13-22, 1986.

[70] G. Ballinger, "Models for intensive longitudinal data," Organ. Res. Methods, vol. 11, pp. 412-414, 2008.

[71] E. Harmon-Jones, "Clarifying the emotive functions of asymmetrical frontal cortical activity", Psychophysiol., vol. 40, pp. 838848, 2003.

[72] M. Quirin, T. Gruber, J. Kuhl and R. Düsing, "Is love right? Prefrontal resting brain asymmetry is related to the affiliation motive," Front. Hum. Neurosci., vol. 7, pp. 1-11, 2013.

[73] D. Schweiger, G. Stemmler, C. Burgdorf and J. Wacker, "Opioid receptor blockade and warmth-liking: effects on interpersonal trust and frontal asymmetry," Soc. Cogn. Affect. Neurosci., vol. 9, pp. 1608-1615, 2014.

[74] N. Ravaja, T. Saari, M. Salminen, J. Laarni and K. Kallinen, "Phasic emotional reactions to video game events: A psychophysiological investigation," Media Psychol., vol. 8, no. 4, pp. 343-367, 2006, doi:10.1207/s1532785xmep0804_2.

[75] N. Ravaja, M. Turpeinen, T. Saari, S. Puttonen and L. Keltikangas-Järvinen, "The psychophysiology of James Bond: phasic emotional responses to violent video game events," Emotion, vol. 8, no. 1, pp. 114-120, 2008, doi:10.1037/1528-3542.8.1.114.

[76] W. van den Hoogen, K. Poels, W. IJsselsteijn and Y. de Kort, "Between challenge and defeat: Repeated player-death and game enjoyment," Media Psychol., vol. 15, pp. 443-459, 2012.

[77] T. Chartrand and J. Bargh, "The chameleon effect: the perception-behavior link and social interaction," J. Pers. Soc. Psychol., vol. 76, no. 6, pp. 893-910, 1999.

[78] A. Todorov and B. Duchaine, "Reading trustworthiness in faces without recognizing faces," Cogn. Neuropsychol., vol. 25, pp. 395-410, 2008.

[79] A. Paulus, M. Rohr, R. Dotsch and D. Wentura, "Positive feeling, negative meaning: Visualizing the mental representations of in-group and out-group smiles," PLoS ONE, vol. 11, no. 3, e0151230, 2016.

[80] J.M. Susskind, D.H. Lee, A. Cusi, R. Feiman, W. Grabski and A.K. Anderson, "Expressing fear enhances sensory acquisition," Nat. Neurosci., vol. 11, pp. 843-850, 2008.

Niklas Ravaja is a professor of social psychology of ICT at the University of Helsinki. He is an expert on emotional and physiological processes during mediated social interaction.

Gary Bente is a professor of psychology at the University of Cologne. His research focuses on nonverbal communication in face-toface and mediated encounters.

Jari Kätsyri is a post-doctoral researcher at Aalto University. He is an expert on emotion research and human-computer interaction.

Mikko Salminen works as a researcher at Aalto University School of Business. His research focuses on emotions in technology-mediated and face-to-face social interaction.

Tapio Takala is a professor of computer science at Aalto University. $\mathrm{He}$ is an expert on computer animation, motion capture and analysis, and embodied/enactive interfaces. 